\title{
Article
}

\section{The Rupture as a Drawing-in of Experience}

\author{
Kay-Jones, Simon
}

Available at http://clok.uclan.ac.uk/29375/

Kay-Jones, Simon ORCID: 0000-0001-5741-3875 (2019) The Rupture as a Drawing-in of Experience. Drawing Research Theory and Practice, 4 (2). pp. 311-325. ISSN 2057-0384

It is advisable to refer to the publisher's version if you intend to cite from the work. http://dx.doi.org/10.1386/drtp_00010_1

For more information about UCLan's research in this area go to http://www.uclan.ac.uk/researchgroups/ and search for < name of research Group>.

For information about Research generally at UCLan please go to http://www.uclan.ac.uk/research/

All outputs in CLoK are protected by Intellectual Property Rights law, including Copyright law. Copyright, IPR and Moral Rights for the works on this site are retained by the individual authors and/or other copyright owners. Terms and conditions for use of this material are defined in the policies page.

\section{CLoK}

Central Lancashire online Knowledge www.clok.uclan.ac.uk

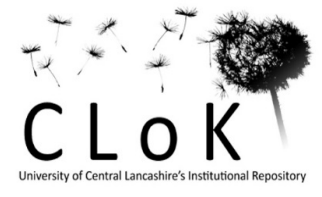




\section{Introduction}

Architectural drawings are particularly helpful, in exploring emergent strategies in drawing as a discursive vehicle. This paper explores the role of student's drawings in-experience throughout the course of a third year architectural design project called 'The Scenographers Chapel. The two sets of images (Figures 1 \& 2) are from a collection of student drawings exploring the rupture as a drawing in of experience.

The Aim of this paper is to position the notion of the rupture as a framework and support structure to drawing in the pursuit of a new theory and emergent terroire of drawing (Figure 3). In order to achieve this the paper speculates the process of drawing to be at the intersection of Material Engagement Theory (MET) as a creative endeavour to assist in exploring the act of rupturing as a potential new theoretical and methodological device.

Traditional educational paradigms have typically centred upon deductive and inductive design strategies with philosophical and ontological positions that from either a positivistic perspective, or a critical rationalist one. Thinking that has emerged out of the shortfalls of a deductive and inductive approach to learning over the last few decades -especially within architectural education- has sought to position itself as a form of either radical learning (Danvers, 2003), or continuous experimenting (Gowan, 1975), (Freire, 1970). Their approach to design has been seen as an experiment in alternative models that lend scientific realism to the chosen project. From particular British traditions, (Ford \& Harris, 2015) in Critical thinking (Davies, 2015) and reflective teaching (Larrivee, 2000), these recent endeavours have sought to co-construct teachers into the social scene of learners (Vygotsky, 1980) (Kolb \& Kolb, 2005), and depart from the material engagement of the phenomenon as a vehicle in itself. This has resulted more recently in material turns (Mara \& Hawk, 2009) (Muller, 2015) and the establishment of critical making projects (Ratto, 2011). All however have never quite managed to compress all of these aspects and acknowledge where they are experienced together in the moment.

The author has therefore been in search of a theory and method that acknowledges the possibility of experiences and the construction of culture as drawn in to the learning moment. A new theory to explore the materiality of drawing that can draw in all aspects related to the subject, object and context of a phenomenon.

The first part of this paper briefly situates MET theory and discusses its relationship to the notion of a rupture and drawing as a material engagement. The second part provides an example for how this is staged in a student led project, providing a method for learning. The concluding part of the paper discusses an emerging theory of drawing summarising the principles underpinning the notion of a rupture in drawing.

\section{Drawing as a Material Engagement}

When we consider the in-process and in-experience of drawing we may find common parallels within recent discourse in Material Engagement Theory (MET theory) (Malafouris, 2008) (Iliopoulos, 2018) (Renfrew, 2004). Indeed MET theory sees drawing and the line of drawing as the psychical and physical. A delineation of mark and the process (Malafouris, 2018). 
And while it might not be, at first analysis, self-evident to explore drawing through its materiality and procedural approach (Overmann \& Wynn, 2018), it does reveal a fertile ground to the role of drawing in developing and delivering learning outcomes to students by exploring drawing in the context of MET theory.

MET theory allows us to view conventional drawing as a compression of the conflation (Malafouris, 2018). Conflation occurs where the brain and the body interact with a wider cultural context. The compression of this conflation for MET theory is drawing in all aspects related to the subject, object and context of a phenomenon. A compression of the ground to which we draw, (ontologically epistemologically, and methodologically) upon three levels of action, object, and meaning (Huiskamp, 2016). This paper argues for a fourth level, that of the experience itself.

The drawing in of experience is then the process towards a conflation of the compression where encounters, such as the action of drawing, the object of drawing and the measure of intent in drawing, conflate as a unified experience at an unseen fourth level. This in MET theory is what Malafouris (2018) refers to as thinging applied to drawing. So the compression of the conflation of drawing is to accept the compression in experience, to conflate where the brain, body and culture are existent (Clark, 2008) and acknowledge where they are experienced together.

The cognitive life of a line that drawing provides for -as Malafouris (2018) points' out- is a realisation of the sentience of the act itself. It connects the subject with external materiality and the culture of experience as it is enacted (Tewes, 2016). It breaks the temporal and episodic approach to drawing of an idea, or mental projection (Ihde \& Malafouris, 2018) within the artist that precedes experience of the materialisation of the drawing (Ingold, 2016). Material engagement is the opposite of what Ingold (2016) posits as a theory of inversion; an artificial schematic set as a distinction between mind and drawing (Rowe \& Koetter, 1978).

For MET theory there is no inversion only compression and conflation as a territorial experience of drawing as Thinging or rather a presence embodied and extended in acts of drawing (Overmann, 2017), that acknowledges the absence of parts yet unrealised of a drawing; Meaning, presenting, remembering. The role of drawing seen in this light is to enable conflation and compression and to offer a vehicle to apprehend the act of this conflation and compression in action, in thinging. The presence and absence provides forth a persistence in drawing that is significant. Drawing operates on different experiential levels and become more than a drawing by the nature of it being drawn.

\section{The Rupture as a Drawing in of Experience: Amplifying the Material Engagement}

MET theory goes further and positions the thinging (drawing in of experiences) as different from thinking. Rather than thinking about things in their absence, things draw with and through things (Malafouris, et al., 2014). The rupture of this thinging is presented here as a method to amplify this process, that may lead to a more temporal experience of drawing out of a new terroire. Rupturing therefore theoretically allows the student to explore the presence and absence of a drawing in unison.

Similar to MET's approach to mind and thinging it can be argued by extension there are no universal things as drawings conceptually, only numerous variants of drawing enacted by 
specific subjects or Actants in specific situations. The process of drawing is not limited to the line that follows the mind but rather the process of drawing in, of experience to the specific situation of investigation (Malafouris, 2010). A plasticity in MET terminology that illustrates a mind becoming through drawing.

\section{Meta-plasticity and the Rupture}

MET refers to this approach as a human cognitive becoming (Malafouris, 2010). Material engagement applied to drawing allows for a form of plasticity in subject and context at varying scales and temporal rhythms to coexist (Kahneman, 2000) in a drawing at anyone moment. Drawing becomes the medium of experience (Pallasmaa, 2005) that evidences the plastic mind drawn into a plastic culture. A drawing of meta-plasticity (Malafouris, 2010), through rupture.

Reframing drawings in this way opens up the full potential of analysis through MET theory to students to investigate a reflective approach to designing, situated within their subject from their chosen context in the construction of a drawing as a point of architecture (Rowe \& Slutsky, 1997). The tension between a presence and absence of their drawing in relation to architecture becomes the scenes for 'cognitive ecology' (Hutchins, 2010) and the drawing evidences a formation of that terroire (a temporal experience) as an Urban Ecology (Rossi, 1982).

The central ambition for the act of drawing in this project responds to the challenge laid down by MET theory, of devising methods for tackling the relational domain as it becomes realised in different contexts of situated action (Malafouris \& Renfrew, 2010). Drawing is an opportunity for the tracing of experience for learning rather than the evidence of learning as an outcome and thereby provides a considered method for approaching relational domains.

Through the process of rupture, this paper positions drawing at the heart of a reframing of the interactions of things and experiences of material agency (Sutton, 2008) and material imagination through the act of drawing. An 'Ecology of mind' (Hutchins, 2010) (Malafouris, 2018) as MET theory refers to it or rather the terroire (Gallagher \& Ransom, 2016) of drawing put forward here. Drawing offers the possibility of a useful methodological approach within the emergent theory of Material Engagement.

\section{Drawing of Process. A Method for Learning}

Students experienced a place as a free play and dérivé within a contemporary city, recording their perceptions, observations and journeys; secondly, they condensed and flattened these experiences by constructing a compositional abstraction and placing these into one continuous drawing as a flat space. By omitting the scale, materiality and shadow from the constructed piece and rupturing their semiotic connection (Beetz, 2016) from the original context, the students brought forth a new ambiguous form, distinct and separate from the original source with particular proportional and compositional dialogues.

Once drawn students went on to rupture the drawing, carving out narratives and actants (Latour, 2007) (Akrich \& Latour, 1992) with the act of rupturing within the flattened (compressed) 
space, eliciting transient and the uncanny states (Trigg, 2012). Student's in-scripted (Bijker \& Law, 2000) compositions to arrive at an ambiguous object either through expressive drawings. Students went onto interpret the rupture as space \& volume; the transient as time \& activity; and uncanny as meaning \& semiotics. Students thereby constructed architecture grounded in the everyday activity; and of the language and meaning of local actants, but also transitioned to an abstract level contained within a body of work that continued to be un-inhabited and ambiguous in form.

\section{Drawing in Making and Experience}

A body of work such as this gives an opportunity to analyse drawing and its importance for those in related fields. The paper speculates the process of drawing to be at the intersection of those MET theories of conflation and compression and with the act of rupturing able to amplify the transient and the uncanny moments of thinging.

The rupture was an act to which students worked towards uncanny and transient states, as a grounded experiential drawing strategy. Such a strategy evidenced the experiences within drawing as bound in a tension of being distinct and connected concurrently, of being ambiguous in their separateness and uncanny in their association with other contexts while at the same time felt as inseparable; a conflation of compression in drawing.

Within the drawings, architectural objects could not sustain themselves without a generated subject and contrarily the subjective meaning of architectural objects could not survive without the object of its consideration. While they emerged together in unison, a rupture was needed for both to be apprehended. An apprehension that was transient to the students and in that transience there was an 'uncanny whole' that emerged. They were glimpses of the Ecology of the Mind (Hutchins, 2010).

The rupture at once separated briefly a subject and its object and at the same time announced the connectivity by the very act of rupturing (Tschumi, 1994). A conflation of the compression of drawing emerged and in the act of rupturing, the form and the subject of the transient and the uncanny became ambiguous. They could be interchanged. By reframing drawing in this context, students were able to reveal or provoke certain ambiguous forms, and uncanny experiences to emerge. The drawing its self was an ambiguous form. It was evidence of metaplasticity and could be described as thinging, with uncanny and ambiguous components.

For the students the driver was not in the making, nor in the production of a drawing itself, but in the intersection between the uncanny and the transient in the act of drawing and the discourse that emerged from that act. In the rupture itself, they became aware of the presence and absence of their work as thinging. The drawings facilitated an all too brief moment where the work invited students to engage with this interlude. To apprehend and work with the moment before the meaning rushed in and the tension was broke. New narratives and actants were in-scripted into the drawing concurrent realities were existent and speculative futures enacted.

These pieces viewed then as an experimentation in semiotic (Beetz, 2016) were a rich seam for discussion. They formed an uncanny whole with the expressed will of being revelatory and provocative to the students. Drawing as it is understood here must be a play on the realization of a thought through making. 
Such educational drawing in experience revealed certain unwritten principles in pedagogy also. Principles, which once analysed partly by the students illuminated a new existential ground for how drawing could be used in architectural projects and its role in the creation of experiences for the realization of a thought through drawing in process.

The images illuminated the core unwritten principle of the rupture, as an integral part to the design strategy, towards thinging. The act of rupturing the drawings then facilitated the understanding of how the current ambiguous form of the drawing, its boundary and its meaning became a driver of culture, and at the same time, the ground to which it sprung, allowing new ideas to emerge. A cognitive life of drawing had occurred.

\section{Conclusions}

Unpacking such grounds and monitoring the success of these strategies was the primary task of this architectural project. The rupture as an architectural artefact laid bare in the drawings, facilitated the creative learning of students with a valuable new methodological approach to reframing existing educational paradigms and emergent theoretical work in material engagement theory applied to drawing.

The rupture as drawing in of experience provided an engagement with the material aspects of experience and an opportunity to put forward specific conclusions to the importance and role of drawing in education. Such a rupture evidenced four distinct aspects of drawing (Figure 3), A. The act of drawing as a means to illuminate fields of learning as distinct paradigms of design strategies; B. The process of drawing as a strategy of architectural work from a structuralist perspective; $\mathrm{C}$. The construction of a drawing process, as a sequentially mapped out procedure of work; and D. The experiencing of drawing in broadening the context to which a multitude of practitioners develop a new Terroire of drawing.

\section{The Act of Drawing: Field}

A specific methodological approach to each drawing project was beneficial to ensure a conceptual rigour to the process of drawing whether implicit or explicit. The work highlighted one particular drawing strategy to which if we take note of the qualitative research paradigms (Denzin \& Lincoln, 1994), can call an example of the Abductive drawing strategy. This involved the construction of drawings grounded in the everyday activity and of the language $\&$ meaning of local Actants from an interpretive constructivist perspective.

Furthermore, we can speculate that 4 drawing strategies are possible in defining a broad categorization of design thinking and drawing process that share similar ontological, epistemological and methodological premises, the inductive, the deductive, and the retroductive. For the purposes of undergraduate design courses, these four broad approaches can be thought of as means to approach a specific issue (1. Strategy) through appropriate lines of inquiry (2. Journey) in a constructed context (3. Terroire). 


\section{The Process of Drawing: Strategy}

Looking again at the critical and cultural theory of drawing specifically within architecture through the prism of drawing strategies in this case the abductive drawing strategy, might allow a radical reframing of the architectural canon and more broadly the drawing praxis of practitioners. Such a strategy has shown to facilitate students moving away from 'what architects make'; their stated intentions and production of architectural artefacts in this case drawings; towards 'what architects do and how they work'. The strategy became a means to develop the student's praxis.

\section{The Construction of Drawing: Journey}

By providing a systematic approach to constructing a journey through drawing, education can nudge the drawer towards the understanding of the context to which they operate the intersection between the physical and the mental within the production of drawing. An understanding, which is at the heart of the material engagement project.

The role of drawing in education should therefore not teach the body of knowledge (types of drawing and its purpose) together with the attainment of skill (mastery in drawing and execution) as distinct, separate canons, but allow for the momentary rupture to be enacted to generate the construction of the drawing. In this instance as a point of departure for architectural discourse, where knowledge and skill are constructed concurrently through the work.

\section{The Experiencing of Drawing: Terroire}

An understanding of a drawing's new terroire in providing and receiving social impacts together with the capacity to have social shaping affects upon wider communities acknowledges the co-evolutionary nature of such terroire and their territorial experience. It treats the context of drawings as a construct and area of interest momentarily captured within the drawing itself, and allows the rupture as a drawing in of further experience.

\section{The Role of Drawing}

These four aspects of drawing and their role in influencing the progression of students in developing a new terroire in drawing are evidence of an emergent theory and methodological approach in using drawing to engage with cultural and architectural conversations, materially. It has wide implications in education and practice.

Material engagement theory, its three levels of action, object and meaning together with the fourth proposed here, that of experience, were enhanced and amplified by the process of rupturing as drawing in of experience. With the process of rupture, this paper positions the act of drawing at the heart of a reframing of interactions, things and experiences through material agency and material imagination. 
Such a rupturing offers a means to tackle the relational domains in MET theory as they become realised in different contexts and situated actions. Rupturing as a method offers the potential for significant and insightful opportunities in understanding the role of drawing and its ability to further MET theory's main aim of establishing humans as thingers (as drawers).

The Paper also puts forward the notion that the role of drawing more broadly may sit along materiality, material turns, matters of agency and its techniques to interact into and through a wider anthropological study of drawing as a comparative study in the materiality of art. The way drawings affect our learning, our thinking and our understanding of culture and matter. 